\title{
Experimental Implementation of a Hybrid Nonlinear Control Design for Magnetostrictive Actuators
}

\author{
William S. Oates ${ }^{1}$, Phillip G. Evans ${ }^{2}$, Ralph C. Smith ${ }^{3}$, and Marcelo J. Dapino ${ }^{4}$ \\ Department of Mechanical Engineering ${ }^{1}$ \\ Florida A\&M/Florida State University \\ Tallahassee, FL 32310-6046 \\ Center for Research in Scientific Computation ${ }^{3}$ \\ Department of Mathematics \\ North Carolina State University \\ Raleigh, NC 27695 \\ Department of Mechanical Engineering ${ }^{2,4}$ \\ Ohio State University \\ Columbus, $\mathrm{OH} 43210$
}

\begin{abstract}
A hybrid nonlinear optimal control design is experimentally implemented on a magnetostrictive Terfenol-D actuator to illustrate enhanced tracking control at relatively high speed. The control design employs a homogenized energy model to quantify rate-dependent nonlinear and hysteretic ferromagnetic switching behavior. The homogenized energy model is incorporated into a finite-dimensional nonlinear optimal control design to directly compensate for the nonlinear and hysteretic magnetostrictive constitutive behavior of the Terfenol-D actuator. Additionally, robustness to operating uncertainties is addressed by incorporating proportional-integral (PI) perturbation feedback around the optimal open

\footnotetext{
${ }^{1}$ Email: woates@eng.fsu.edu, Telephone: (850) 410-6190

${ }^{2}$ Email: evans.895@osu.edu, Telephone: (614) 247-7480

${ }^{3}$ Email: rsmith@eos.ncsu.edu, Telephone: (919) 515-7552

${ }^{4}$ Email: dapino.1@osu.edu, Telephone: (614) 688-3689
} 
loop response. Experimental results illustrate significant improvements in tracking control in comparison to PI control. Accurate displacement tracking is achieved for sinusoidal reference displacements at frequencies up to $1 \mathrm{kHz}$ using the hybrid nonlinear control design whereas tracking errors become significant for the PI controller for frequencies equal to or greater than $500 \mathrm{~Hz}$.

Keywords: nonlinear optimal tracking, magnetostrictive, rate-dependence, perturbation control

\section{Introduction}

The role of smart materials continues to be a critical part of technology development in many biomedical, automotive, aerospace, and industrial applications. These materials provide advantages in applications where large forces and small displacements are desired over a broad frequency range with high precision. The solid state characteristics of these materials provide compact actuators which are critical in applications where size and weight are important. A large number of these applications employ piezoelectric or magnetostrictive materials which respectively possess electric or magnetic field induced displacement and force. For example, single crystal ferroelectric relaxors (PMN-PT or PZNPT) have provided significant advances in sonar transducer applications due to their efficiency and relatively high strain behavior $(\leq 1 \%)[1]$. Additionally ferroelectric lead zirconate titanate $(\mathrm{PZT})$ has been successfully implemented in commercial nanopositioning stages for controlling the position of material specimens for probing atomic structures using atomic force microscopy and scanning probe microscopy [2]. The robustness of magnetostrictive terbium-iron-dysprosium (Terfenol-D) actuators has provided reliable actuator designs for several applications including precisely machined out-ofround piston heads by effectively controlling the cutting tool position [3].

Although ferroic materials have been successfully implemented in a number of applications, limitations associated with nonlinear and hysteretic material behavior have presented challenges in developing high performance actuation response over a broad frequency range. The nonlinear and hysteretic material behavior is primarily due to the reorientation of local electric or magnetic variants that align with the applied electric or magnetic fields. Moderate to large field levels can induce $0.1 \%$ strain in polycrystalline PZT [4] and up to $6 \%$ strain in single crystal ferromagnetic shape memory alloys [5]; at these field levels, obtaining accurate and precise control is greatly complicated by nonlinearities and magnetic hysteresis. This has motivated research in developing new control designs that can effectively 
compensate for nonlinearities and hysteresis induced by ferroelectric or ferromagnetic switching while still providing accurate forces or displacements over a broad frequency range.

Two general strategies are typically considered when developing a control design to compensate for nonlinearities and hysteresis. One approach is to implement a nonlinear inverse compensator which approximately linearizes the constitutive behavior so that linear control methods can be employed [6-11]. This approach provides the ability to implement well-developed linear control laws. It can also be advantageous in applications where an unknown disturbance load is present or the reference signal is not known well in advance; however, this advantage is only realized if the constitutive model is efficient enough to be inverted in real-time. The second strategy typically considered entails direct incorporation of the material model into the control design so that the nonlinear control input is directly determined. This circumvents issues associated with computing the constitutive inverse law, but introduces challenges in identifying robust numerical algorithms that can achieve convergence efficiently.

Both of these control designs require an efficient and accurate constitutive model that can predict the nonlinear and hysteretic ferroic switching behavior. Preisach models are often considered for quantifying nonlinear and hysteretic material behavior in ferroic materials and have also been employed in model-based control designs: see $[8,12]$ for examples. These models have the disadvantage of requiring a large number of unphysical parameters to predict minor loop hysteresis. In the analysis presented here, a ferroic homogenized energy model is implemented which utilizes fundamental energy relations at the mesoscopic or lattice length scale to quantify macroscopic constitutive behavior in ferroic materials. The model utilizes a multi-scale approach which relates local material inhomogeneities using a stochastic framework to predict macroscopic material behavior [13-17]. This modeling framework has been successful in accurately quantifying rate-dependent major and minor hysteresis loops in several ferroelectric, magnetostrictive and shape memory alloy compositions; see [17] for a review. The stochastic modeling approach utilizes general densities which can be fit to experimental results. This improves model prediction, which is critical in model-based control designs so that the amount of feedback required to achieve a specified performance criteria is reduced.

The second strategy in developing a model-based nonlinear control design is presented here where the constitutive law is directly incorporated into an optimal control design. This approach is shown 
to improve tracking control accuracy for a magnetostrictive transducer at frequencies up to $1 \mathrm{kHz}$. The authors are only aware of one other model-based control design successfully implemented experimentally on a similar magnetostrictive actuator [8]. In their analysis, a Preisach-based nonlinear inverse compensator was employed. The reference displacement was limited to aperiodic signals centered around $30 \mathrm{~Hz}$ and tracking control was improved in comparison to proportional control. In the analysis presented here, comparisons between the nonlinear optimal control design and classical proportional-integral (PI) control are conducted. It is demonstrated that PI tracking control performance begins to degrade for sinusoidal reference displacements with frequencies at or above $500 \mathrm{~Hz}$. The bandwidth of the actuator is improved by directly incorporating the constitutive behavior within the control design. Reasonable tracking control is achieved for frequencies up to at least $1 \mathrm{kHz}$.

The experimental analysis presented here utilizes a nonlinear control design previously analyzed numerically for controlling a magnetostrictive transducer operating in current control mode $[18,19]$. The model is extended to include voltage control to accommodate the experimental set-up and is validated over a broader range of frequencies $(100 \mathrm{~Hz}$ to $1000 \mathrm{~Hz})$ than previous numerical analyses. In Section 2, the experimental set-up is described. In Section 3, the constitutive model and dynamic model are presented and compared to open loop actuator characterizations. In Section 4, the nonlinear control design is presented and compared to classic proportional-integral (PI) control to identify operating regimes where the nonlinear control design provides enhanced performance. Section 5 includes discussion and concluding remarks.

\section{Experimental Implementation}

The validation of the proposed control method was performed on an Etrema Products, Inc., magnetostrictive Terfenol-D actuator model MFR OTY77. The actuator employs a Terfenol-D rod 12.5 $\mathrm{mm}$ in diameter by $100 \mathrm{~mm}$ in length which is subjected to a 10-14 MPa preload and $\sim 40 \mathrm{kA} / \mathrm{m}$ magnetic field bias from a permanent magnetic. The drive coil rating is $6.2 \mathrm{kA} / \mathrm{m} \cdot \mathrm{A}$ with a 3.4 Arms

limit. Figure 1 illustrates the basic internal components in the Etrema actuator which includes a Terfenol-D rod, a compression bolt and spring washer to preload the rod, a surrounding wound wire solenoid and permanent magnet.

The drive voltage is generated by a 16-bit digital-to-analog converter (DAC) on a dSPACE DS1104 
controller board which has an output range of $10 \mathrm{~V}$ and $>80 \mathrm{~dB}$ signal-to-noise ratio. The drive voltage is amplified by an AE Techron LVC 5050 linear amplifier set to a gain of $17 \mathrm{~V} / \mathrm{V}$. The bandwidth of the amplifier is $20 \mathrm{kHz}$.

Reported data includes the DS1104 drive voltage, actuator current, and actuator strain. The current supplied to the actuator is measured by the Techron's current monitor which outputs $1 \mathrm{~V}$ for every 3 amperes of current. The current monitor signal is sampled using the 16-bit analog-to-digital (ADC) on the DS1104 board. The strain measurement is simultaneously sampled with the same ADC from a Lion Precision capacitive sensor (PX405JTC probe with DMT10R driver) with a sensitivity of $2.5 \mathrm{~m} / \mathrm{V}$ and bandwidth of $12.5 \mathrm{kHz}$. An Omega Engineering, Inc. signal amplifier model OMNI-AMP III DC with a gain of $10 \mathrm{~V} / \mathrm{V}$ and a bandwidth of $10 \mathrm{kHz}$ is used to match the dynamic range of the strain measurement with the range of the ADC.

Tests were setup as block diagrams using Mathworks Simulink. The block diagrams were then compiled and downloaded to the DS1104 controller using Real Time Workshop, also by Mathworks. Data was acquired using ControlDesk by dSPACE with a sample frequency of $10 \mathrm{kHz}$.

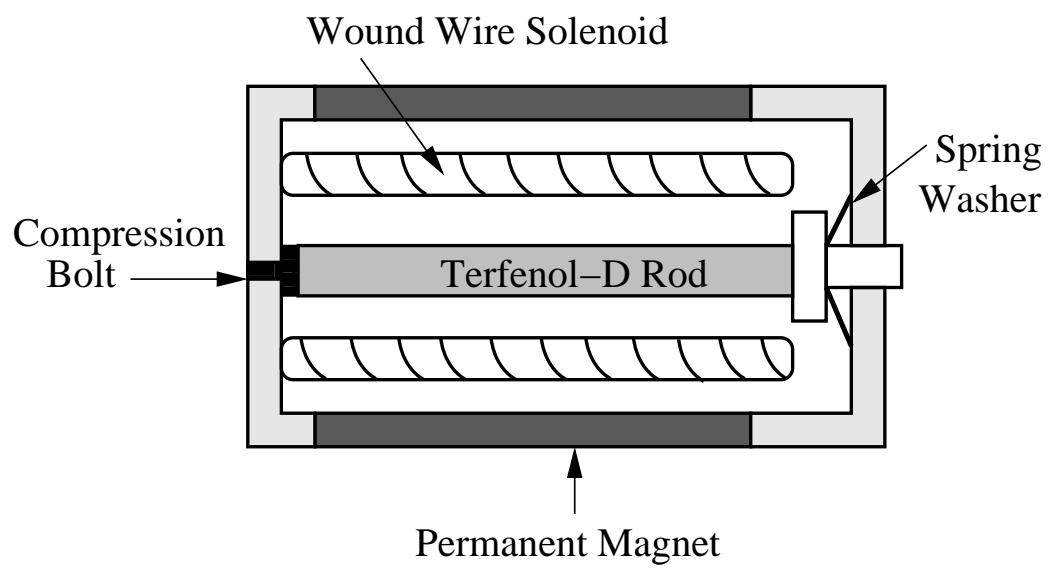

Figure 1: Schematic of the Terfenol-D actuator used in the control experiments. 


\section{Model Development}

The modeling framework used in implementing the control design incorporates magnetostrictive nonlinearities and hysteresis to predict actuation. The ferromagnetic switching behavior is modeled using a homogenized energy model that is based on previous work described in detail in [13-17,

20]. Here, only key equations are given to motivate the implementation of the constitutive model in the structural dynamic model and control design. The homogenized energy model is formulated using an applied magnetic field; however, the power amplifier used in the control experiments uses voltage control. Although a hardware modification can be employed to run the power amplifier in current control, the homogenized energy model was extended to relate voltage to current to simplify experimental implementation. This approach results in directly determining the nonlinear voltage control input from the homogenized energy model, structural dynamic relations and optimal control design.

In addition to quantifying the ferromagnetic switching behavior, the structural dynamics of the actuator is quantified using a lumped parameter model. Although a distributed weak PDE formulation can be employed to incorporate spatial dependence along the actuator length, the operating frequencies considered are below resonance; therefore, a lumped parameter model reasonably approximates the structural dynamics for the sinusoidal reference displacement signals used in validating the control design. For more general reference displacements, such as a step input that excites higher order harmonics, a finite element model may be necessary. Finite element models can be directly incorporated into the model and control design as discussed in [17-19].

First the homogenized energy model is briefly summarized. Second, the equations associated with the structural dynamic model are summarized to quantify displacements predicted by the homogenized energy model for a magnetic field input. The nonlinear electrical impedance of the amplifier-actuator system is then modeled to relate the magnetic field to the voltage input for controlling the Terfenol-D actuator using voltage control. Lastly, the homogenized energy model is compared to experimental results.

\subsection{Rate-Dependent Ferromagnetic Homogenized Energy Model}

The homogenized energy model is based on an energy description at the mesoscopic or lattice 
length scale. This local energy formulation is used to predict macroscopic constitutive behavior using a stochastic representation of material inhomogeneities relating the nucleation and growth of local ferromagnetic domains to macroscopic actuator displacements.

The constitutive law used in modeling the Terfenol-D rod actuator in Figure 1 focuses on the one dimensional case in the direction of uniaxial loading parallel to the rod length. In this case, material coefficients and field quantitites have been reduced to scalar coefficients or distributed variables in the direction of loading. The Gibbs energy at the mesoscopic length scale is then

$$
G(M, T)=\Psi(M, T)-\mu_{0} H M
$$

where $\Psi(M, T)$ is the Helmholtz energy detailed in [17], $T$ is temperature, $H$ is the magnetic field, and $M$ is the magnetization. The one-dimensional Helmholtz energy function is a double-well potential below the Curie point $T_{c}$ which gives rise to stable spontaneous magnetization with equal magnitude in the positive and negative directions.

The effects of rate-dependent hysteresis under applied fields are often present and must be included in the constitutive model. This effect is modeled using the Boltzmann relation

$$
\mu(G)=C e^{-G V / k T}
$$

which quantifies the probability $\mu$ of achieving an energy level $G$. Here $k T / V$ is the relative thermal energy where $V$ is a representative volume element at the mesoscopic length scale, $k$ is Boltzmann's constant, and the constant $C$ is specified to ensure integration to unity.

The Boltzmann relation gives rise to the local expected values

$$
\left\langle M_{+}\right\rangle=\int_{M_{I}}^{\infty} M \mu(G) d M \quad, \quad\left\langle M_{-}\right\rangle=\int_{-\infty}^{-M_{I}} M \mu(G) d M
$$

of the magnetization associated with positively and negatively oriented dipoles, respectively. Here $\pm M_{I}$ are the positive and negative inflection points in the Helmholtz energy definition.

The local magnetization variants are defined by a volume fraction of variants $x_{+}$and $x_{-}$having positive and negative orientations, respectively. The conservation relation $x_{-}+x_{+}=1$ must hold for 
the volume fraction of magnetization variants. The kinetic equations govern the evolution of variants that switch. The rate-dependent behavior is determined by a set of transition likelihoods that define the probabilities that magnetization variants switch into negative or positive directions-more details can be found in $[15-17,21]$.

The resulting local average magnetization is quantified by the relation

$$
\bar{M}=x_{+}\left\langle M_{+}\right\rangle+x_{-}\left\langle M_{-}\right\rangle .
$$

The macroscopic polarization is computed from the distribution of local variants from the relation

$$
[M(H)](t)=\int_{-\infty}^{\infty} \int_{0}^{\infty} \nu\left(H_{c}, H_{I}\right)\left[\bar{M}\left(H+H_{I} ; H_{c}, \xi\right)\right](t) d H_{I} d H_{c}
$$

where $\nu\left(H_{c}, H_{I}\right)$ denotes the distribution of coercive fields $\left(H_{c}\right)$, interaction fields $\left(H_{I}\right)$ and $\xi$ represents the initial distribution of the local variants.

The densities can often be modeled as lognormal or normal distributions; however, when more accurate model predictions are critical such as in the case where precision control is desired, a general density can be fit to data. A general density is used in the present analysis and the values are determined by employing a parameter optimization technique to obtain sufficient model accuracy in predicting rate-dependent hysteresis. Details describing techniques to identify general densities can be found in $[16,17]$.

Once the macroscopic magnetization is quantified by (5), the forces generated by the magnetostrictive actuator must be quantified for implementation within the control design. This is provided by the constitutive law

$$
\sigma=Y^{M} \varepsilon+c_{D} \dot{\varepsilon}-a_{1}\left(M(H)-M^{r}\right)-a_{2}\left(M(H)-M^{r}\right)^{2}
$$

representing uniaxial stress in the magnetostrictive actuator. The effective properties of the actuator include $Y^{M}$ as the elastic modulus at constant magnetization, $c_{D}$ as the Kelvin-Voigt damping parameter, $\varepsilon$ as the linear strain component in the direction of loading, $a_{1}$ as the piezomagnetic coefficient and $a_{2}$ as the magnetostrictive coefficient. The time rate of change of the strain is denoted by $\dot{\varepsilon}$. It is assumed that stress fields are limited to the linear elastic regime where ferroelastic switching 
is negligible. The magnetization $M(H)$ is computed using (5) where $M^{r}$ is the initial macroscopic remanent state of the material. The material parameters associated with the homogenized energy model are given in Table 1. These parameters were identified from the experimental results presented in Section 3.4 using parameter optimization techniques detailed in [17]. Note that these parameters are not unique since induction was not measured. The parameter optimization was conducted using experimental displacement measurements and compared to the current applied to the solenoid in the magnetostrictive actuator. This identification procedure requires implementing the structural model described in the following section.

\subsection{Structural Model}

The constitutive relations given by (5) and (6) are used to develop a system model that quantifies forces and displacements when a magnetic field or stress is applied to the magnetostrictive actuator. The partial differential equation (PDE) model is first given and then formulated as a lumped parameter ordinary differential equation (ODE). The effective stiffness, mass and damping factor are determined from the parameter optimization which is based on the structural dynamics of the Terfenol-D actuator and the damped oscillator used in preloading the actuator. A simple schematic of this configuration is illustrated in Figure 2 .

A balance of forces gives $[17,22]$

Table 1: Parameters employed in the homogenized energy model. $\chi_{m}$ is the magnetic susceptibility. A thermal energy parameter, $\gamma=\frac{V}{k T}$, has been used where $k$ is Boltzmann's constant, $V$ is a local representative volume element and $T$ is the temperature. $M^{s}$ is the local remanent magnetization and $\tau$ is the time constant, see [17] for details.

\begin{tabular}{c}
$\chi_{m}=3.3$ \\
$\gamma=1.23 \times 10^{8} \mathrm{~ms}^{2} / \mathrm{kg}$ \\
$M^{s}=190 \mathrm{kA} / \mathrm{m}$ \\
$\tau=1.15 \times 10^{-4} \mathrm{~s}$ \\
\hline
\end{tabular}




$$
\rho A \frac{\partial^{2} w}{\partial t^{2}}=\frac{\partial N_{t o t}}{\partial x}
$$

where the density of the actuator is denoted by $\rho$, the cross-section area is $A$ and the displacement is denoted by $w$. The total force $N_{t o t}$ acting on the actuator is

$$
N_{t o t}(t, x)=Y^{M} A \frac{\partial w}{\partial x}+c_{D} A \frac{\partial^{2} w}{\partial x \partial t}+F_{m}(H)
$$

where the elastic restoring force is given by the first term on the right hand side of the equation and Kelvin-Voigt damping is incorporated in the second term. The model focuses on relative displacements from the preloaded reference state. The linear elastic strain component in the direction of loading is defined by $\varepsilon=\frac{\partial w}{\partial x}$. The coupling force $F_{m}$ represents forces generated by the applied field where

$$
F_{m}(H)=A\left[a_{1}\left(M(H)-M^{r}\right)+a_{2}\left(M(H)-M^{r}\right)^{2}\right]
$$

and the hysteretic and nonlinear $H-M$ relation is specified by (5).

As illustrated in Figure 2, the boundary conditions are defined by a zero displacement at $x=0$ and the balance of forces at $x=\ell$ yields

$$
N_{t o t}(t, \ell)=-k_{L} w(t, \ell)-c_{L} \frac{\partial w}{\partial t}(t, \ell)-m_{L} \frac{\partial^{2} w}{\partial t^{2}}(t, \ell) .
$$

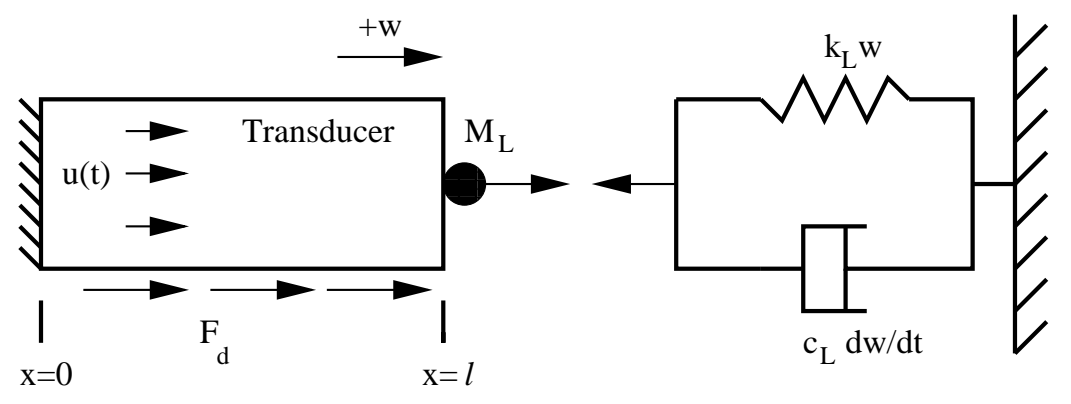

Figure 2: Magnetostrictive actuator with damped oscillator used to quantify loads under a time varying magnetic field. Disturbance forces along the actuator are given by $F_{d}$ and the control input is $u(t)$. 
Table 2: Model parameters for the magnetostrictive actuator and damped oscillator. The parameter optimization identified the magnetostrictive coefficient $a_{2}$ to be zero for the operating regime considered.

\begin{tabular}{l|l}
$\bar{k}=1.96 \times 10^{7} \mathrm{~N} / \mathrm{m}$ & $\bar{m}=.013 \mathrm{~kg}$ \\
$a_{1}=3275 \mathrm{~N} / \mathrm{Am}$ & $\bar{c}_{D}=2.3 \times 10^{3} \mathrm{Ns} / \mathrm{m}$ \\
$a_{2}=0 \mathrm{Nm}^{2} / \mathrm{A}^{2}$ & $A=1.27 \times 10^{-4} \mathrm{~m}^{2}$ \\
\hline
\end{tabular}

The initial conditions are $w(0, x)=0$ and $\frac{\partial w}{\partial x}(0, x)=0$.

\subsubsection{Approximation Method}

The second order differential equation given by (7) with boundary conditions (10) is rewritten in the form

$$
\bar{m} \ddot{w}+\bar{c}_{D} \dot{w}+\bar{k} w=F_{m}(H)+F_{d}
$$

where $\bar{m}, \bar{c}_{D}$ and $\bar{k}$ denote the effective mass, damping and stiffness coefficients, respectively. These parameters represent the effective structural dynamic coefficients for the Terfenol-D rod and damped oscillator as given by

$$
\begin{aligned}
& \bar{m}=\rho A \ell+m_{L} \\
& \bar{c}_{D}=\frac{c_{D} A}{\ell}+c_{L} \\
& \bar{k}=\frac{Y^{M} A}{\ell}+k_{L}
\end{aligned}
$$

Model parameters associated with the magnetostrictive actuator and damped oscillator used in the control design are given in Table 2 .

For control implementation, (11) can be reformulated as a first order system

$$
\begin{aligned}
& \dot{\mathbf{x}}(t)=\mathbf{A} \mathbf{x}(t)+[\mathbf{B}(u)](t)+\mathbf{G}(t) \\
& \mathbf{x}(0)=\mathbf{x}_{\mathbf{0}} \\
& y(t)=\mathbf{C x}(t)
\end{aligned}
$$


where $\mathbf{x}(t)=[w, \dot{w}]^{T}$. The matrix $\mathbf{A}$ incorporates the mass, damping and stiffness matrices given in $(11)$ and $[\mathbf{B}(u)](t)$ includes the nonlinear input where $u(t)$ is defined as the magnetic field. The disturbances are denoted by the matrix $\mathbf{G}(t)$. These matrices are given below in (14). The initial conditions are defined by $\mathbf{x}_{\mathbf{0}}$. The output of the system $y(t)$ is a function of the system states according to the matrix $\mathbf{C}=[1,0]$ where only the displacement at the end of the actuator is measurable.

$$
\mathbf{A}=\left[\begin{array}{cc}
0 & 1 \\
-\bar{k} / \bar{m} & -\bar{c}_{D} / \bar{m}
\end{array}\right] \quad, \quad \mathbf{B}(u)=F_{m}(u)\left[\begin{array}{c}
0 \\
1 / \bar{m}
\end{array}\right] \quad, \quad \mathbf{G}(t)=F_{d}(t)\left[\begin{array}{l}
0 \\
1
\end{array}\right]
$$

Predictions of the constitutive behavior at multiple frequencies using (13) is illustrated in Figure 3 and compared to experimental results. A reasonable estimation of rate-dependent hysteresis is achieved over the frequency range $100 \mathrm{~Hz}$ to $500 \mathrm{~Hz}$ using the parameters in Tables 1 and 2. However, voltage control is used in the control experiments, therefore the model is extended to include nonlinear electrical impedance relations to obtain a model that can quantify the nonlinear voltage control input for experimental implementation.

\subsection{Nonlinear Current-Voltage Relations}

A lumped-circuit model is used to relate the input voltage to the magnetic field applied to the Terfenol-D actuator. A linear power amplifier used in the experiments and the nonlinear impedance associated with the electrical part of the system is assumed to be related to the ferromagnetic switching in the Terfenol-D actuator. This can be modeled using a resistor in series with a nonlinear inductor (i.e., the Terfernol-D rod) and a voltage source that has been amplified by the linear power amplifier.

The nonlinear inductance is quantified by coupling the homogenized energy model with the lumpedelectric circuit model. The first-order nonlinear ODE for a resistor in series with a nonlinear inductor is

$$
\begin{aligned}
& L(M) \frac{d i(t)}{d t}+R i(t)=V(t) \\
& i(0)=i_{0}
\end{aligned}
$$

where $L(M)$ is the inductance written as a function of magnetization, $i$ is the current, $V$ is the voltage input, $R$ is the resistance and the initial conditions are defined by $i_{0}$. 
The magnetic field $H$ applied to the Terfenol-D rod is related to the current by the relation

$$
H=\frac{N}{\ell} i
$$

where $\ell$ is the actuator length and $N$ is the number of coils in the solenoid. Here, the tangential magnetic field on the surface of the Terfenol-D rod is assumed to fully penetrate the rod cross-section area over the frequency range considered. This assumes the effects of eddy current losses are negligible.

The inductance can be related to the magnetic permeability and the wound wire solenoid. From classic electromagnetics [23], the inductance describes the self-induced emf which is proportional to the time rate of change of current. The inductance can therefore be written as

$$
L(M)=\frac{N^{2}}{\ell} \frac{d \Phi_{m}(M)}{d H}
$$

where $\mathrm{N}$ is the number of turns in the solenoid and $\Phi_{m}$ is the flux. The flux is related to the induction through the relation

$$
\Phi_{m}=\int_{A} \mathbf{B} \cdot d \mathbf{A}
$$

where $\mathbf{B}$ is the induction and $\mathbf{A}$ is the surface. Since eddy currents in the Terfenol-D actuator have been neglected and if rod end effects are neglected, the flux can be defined by

$$
\Phi_{m}=B A
$$

for the induction component $B$ parallel to the rod length and $A$ is the cross sectional area.

The nonlinear inductance can be determined by including changes in the remanent magnetization with respect to the change in field given in (17). This can be described by representing the magnetization as a superposition of a linear term and the rate-dependent nonlinear and hysteretic term associated with the change in remanent magnetization. This is considered at the macroscopic length scale by writing (5) as

$$
[M(H)](t)=\chi_{m} H(t)+\left[M^{r}(H)\right](t)
$$


where $\chi_{m}=\mu_{0}\left(1+\chi_{m}\right)$ is the macroscopic magnetic susceptibility and $M^{r}(H)$ is the remanent magnetization. The induction relation $B=\mu_{0}(H+M)$ can then be written as

$$
[B(H)]](t)=\mu_{0}\left(1+\chi_{m}\right) H(t)+\mu_{0}\left[M^{r}(H)\right](t)
$$

where $\mu_{0}=4 \pi \times 10^{-7} \mathrm{~Wb} / \mathrm{Am}$ is the permeability of free space.

The nonlinear induction is then

$$
L(M)=\frac{N^{2} A}{\ell} \frac{d B}{d H}=\frac{N^{2} A}{\ell}\left(\mu_{m}+\mu_{o} \frac{d M^{r}}{d H}\right)
$$

where $\mu_{m}$ is the relative permeability of the material. Note that this relation simplifies to the classic linear inductance relation for a wound wire solenoid when the remanent magnetization is constant. Whereas this approach is expected to provide a relation for nonlinear inductance, $H-B$ data is not available from the Terfenol-D actuator which requires estimating the inductance. A fitting parameter is introduced according to

$$
L(M)=N \frac{d B}{d i} \simeq K_{L} \frac{d y}{d i}
$$

where $y$ is the Terfenol-D actuator displacement determined from (13) and $K_{L}$ is quantified from the experimental results using voltage vs. current data and current vs. strain data. $K_{L}$ was quantified at $100 \mathrm{~Hz}$ and held fixed for all other frequencies considered in the model-based control design. The value that was quantified from the experiments was $K_{L}=2 \times 10^{5} \mathrm{Vs} / \mathrm{m}$ which was used in the model and compared to experiment results presented in Section 3.4. The nonlinear model is also compared to a linearized lumped circuit model where the remanent magnetization is assumed constant. When the inductance is linearized, the model prediction is reasonable in regimes of positive current for the given frequency range. When the current is negative, more ferromagnetic switching occurs which leads to better model predictions using the nonlinear inductance relation. Therefore, the nonlinear model is used in determining the nonlinear voltage control input.

\subsection{Actuator Characterization}

Characterization of the actuator was done using open loop sinusoidal drive voltages at 100, 200, 300 and $500 \mathrm{~Hz}$. Each data set was initiated by a half-cycle of a $1 \mathrm{~Hz}$ sine wave with an amplitude of $1 \mathrm{~V}$ 
to the amplifier-actuator system; this corresponds to a current of $4.6 \mathrm{~A}$. After the initial magnetization half-cycle, sinusoidal voltage signals at frequencies 100, 200,300, and $500 \mathrm{~Hz}$ were used to drive the Terfenol-D actuator. The peak-to-peak sinusoidal voltage inputs were adjusted to achieve minor loops extending $60 \mu \mathrm{m}$. Strain-current minor loops are illustrated in Figure 3 for the frequencies 100, 200, 300, and $500 \mathrm{~Hz}$. The corresponding current-voltage loops are illustrated in Figure 4. The data given in these figures are compared to model predictions using the homogenized energy model and lumped electric circuit model discussed in the previous sections.

\section{Control Design}

To provide a metric of comparison for the hybrid nonlinear control design, we compare its tracking performance to a proportional integral (PI) control design. First the control gains selected for the PI controller are discussed and experimental results describing the closed loop dynamics of the PI controller are presented. Second, the fundamental equations describing the nonlinear control design and PI perturbation feedback are given. This follows previous theoretical and numerical analyses of the hybrid nonlinear control design for ferromagnetic actuators [18, 19,24]; details regarding the algorithm formulation can be found in these citations. Previous numerical analysis focused on quantifying a magnetic field control signal and did not consider the electrical impedance of the amplifier-actuator system. The inclusion of the voltage-current dynamics presented in Section 3.3 is discussed in this section to illustrate how the open loop nonlinear voltage control signal is implemented experimentally.

\subsection{Proportional Integral Control Design}

To facilitate the design of the PI controller, the transfer function of the open loop amplifieractuator system in the near linear regime was measured using a swept sine voltage input from the DS1104 controller board where the output was the rod tip displacement; see Figure 5.

The PI controller was designed using the form

$$
D(s)=K_{P} \frac{s+K_{I} / K_{P}}{s} .
$$

where $K_{P}$ is the proportional gain and $K_{I}$ is the integral gain. The gain $K_{P}$ was chosen to move the mechanical resonance peak observed at $4 \mathrm{kHz}$ below $0 \mathrm{~dB}$. The ratio $K_{P} / K_{I}$ was chosen so that the 


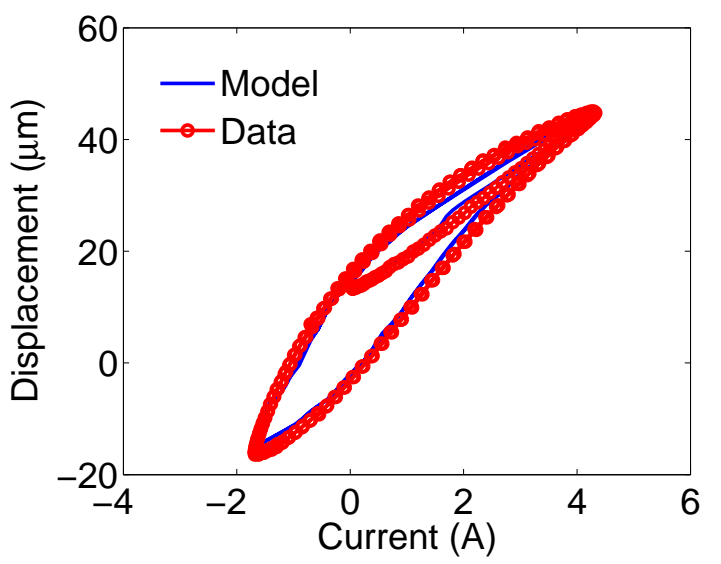

(a)

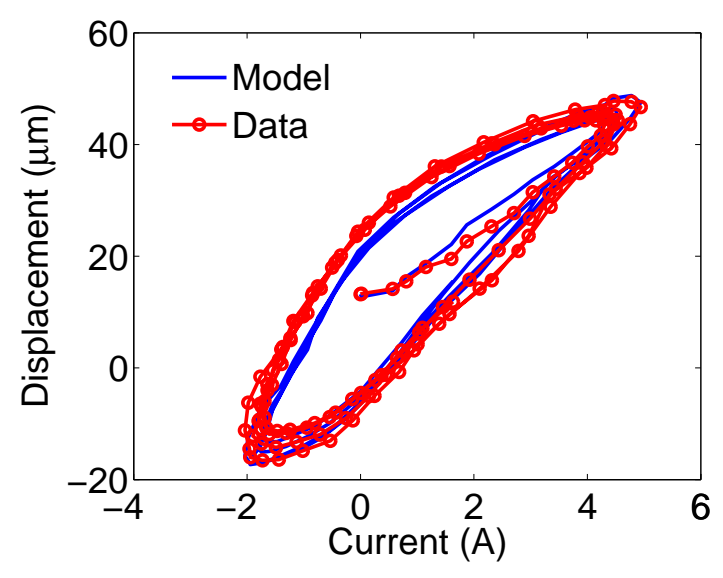

(c)

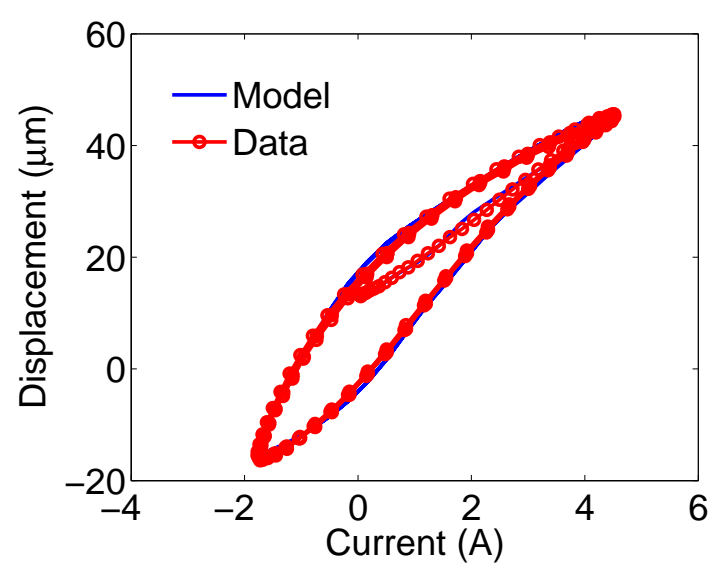

(b)

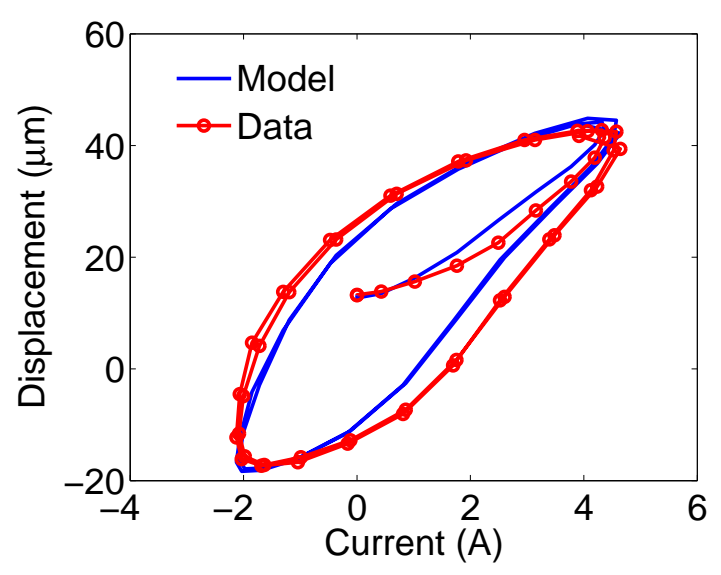

(d)

Figure 3: Rate-dependent constitutive data and comparison to the homogenized energy model described in Section 3. The frequencies tested and fit to the model were (a) $100 \mathrm{~Hz}$ (b) $200 \mathrm{~Hz}$ (c) $300 \mathrm{~Hz}$ and (d) $500 \mathrm{~Hz}$.

phase lag occurred well below the open loop cross-over frequency. Gains of $K_{P}=2 \times 10^{-2} \mathrm{~V} / \mathrm{ppm}$ and $K_{P} / K_{I}=30 \mathrm{~s}$ were chosen. The PI controller boosts the low frequency gain to reduce the steady-state error at the expense of the low frequency phase as illustrated in Figure 6(a). The open loop controller-amplifier-actuator transfer function was measured using a swept sine controller input to determine the stability margins; see Figure $6(\mathrm{~b})$. The phase margin is $45^{\circ}$ and the gain margin is 2. The resulting closed loop bandwidth for PI control is $1140 \mathrm{~Hz}$; see Figure 7. 


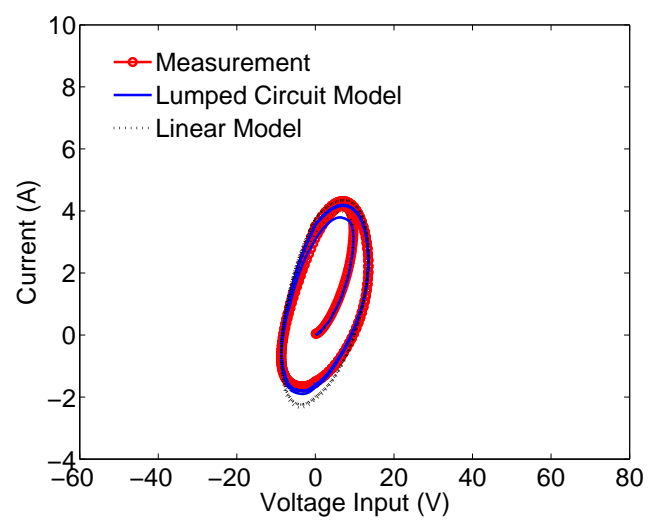

(a)

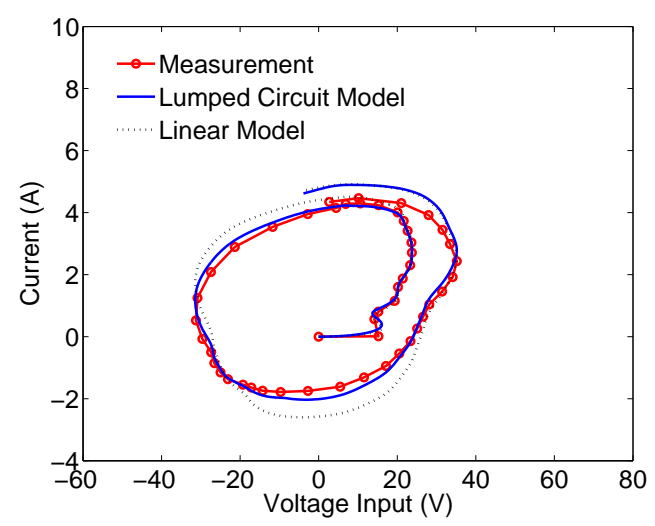

(c)

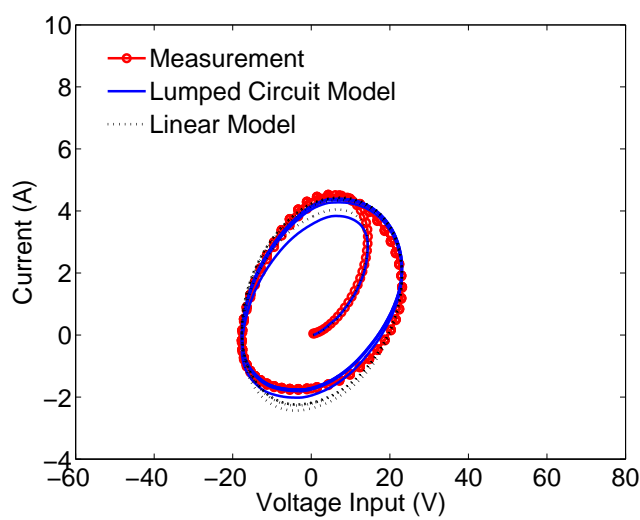

(b)

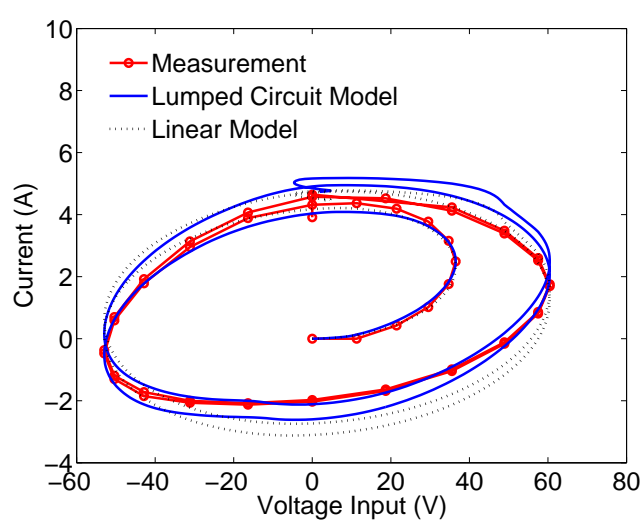

(d)

Figure 4: Current-voltage behavior of the amplifier-actuator system. The data is compared to a linear and nonlinear inductor-resistor lumped circuit model discussed in Section 3.3. The frequencies correspond to the data in Figure 3 where (a) $100 \mathrm{~Hz}$ (b) $200 \mathrm{~Hz}$ (c) $300 \mathrm{~Hz}$ and (d) $500 \mathrm{~Hz}$.

\subsection{Nonlinear Optimal Tracking Control Design}

We summarize here the formulation of a finite-dimensional nonlinear optimal tracking control design where an open loop control signal is computed off-line. To improve robustness to operating uncertainties, perturbation feedback using PI control is implemented. The development of the hybrid nonlinear optimal tracking control design follows previous numerical analyses focused on hysteresis of magnetostrictive actuators for vibration attenuation of beam and plate structures and tracking 

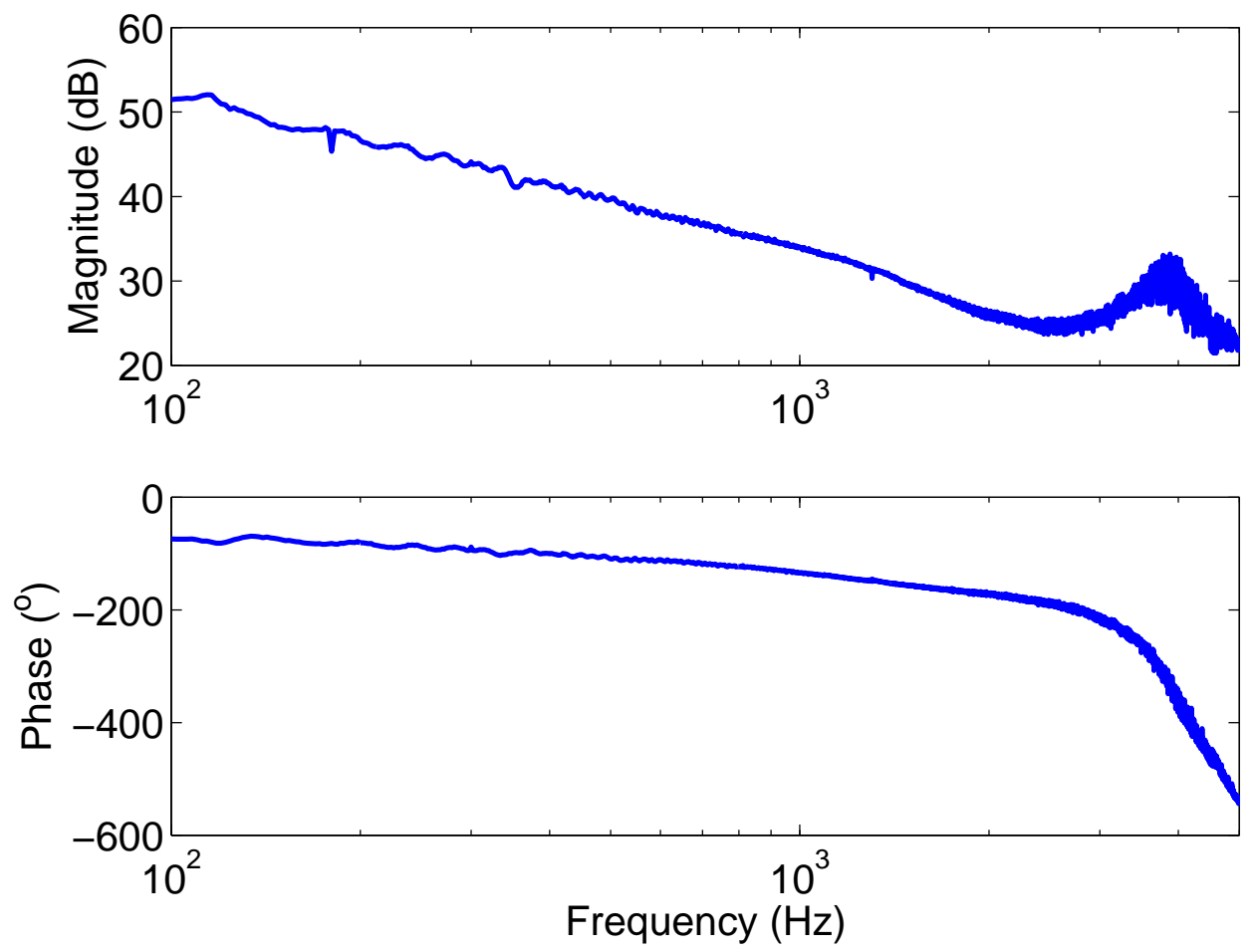

Figure 5: Open loop frequency response for the Terfenol-D actuator in the near-linear operating regime. The input is voltage to the wound wire solenoid and the output is the rod tip displacement.

control of rod structures $[18,19,24]$. We summarize here key equations associated with nonlinear optimal tracking control and perturbation feedback.

Optimal tracking control employs a cost functional to determine the optimal control input. The cost functional

$$
\bar{J}=\frac{1}{2}\left(\mathbf{C x}\left(t_{f}\right)-r\left(t_{f}\right)\right)^{T} P\left(\mathbf{C x}\left(t_{f}\right)-r\left(t_{f}\right)\right)+\int_{t_{0}}^{t_{f}}\left[\mathcal{H}-\lambda^{T}(t) \dot{\mathbf{x}}(t)\right] d t
$$

penalizes the control input and the error between the Terfenol-D actuator displacement and the prescribed displacement where $P$ penalizes large terminal values on the tracking error, $\mathcal{H}$ is the Hamiltonian, and $\lambda(t)$ is a set of Lagrange multipliers. The Hamiltonian is 

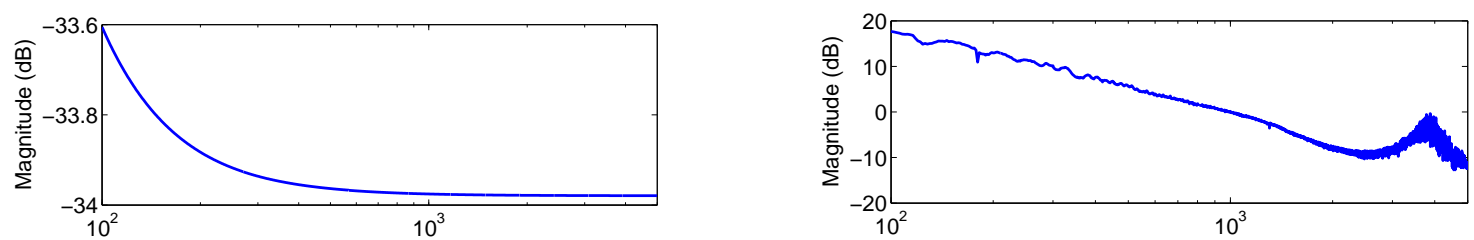

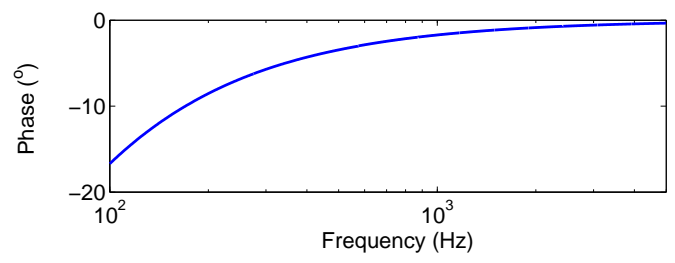

(a)

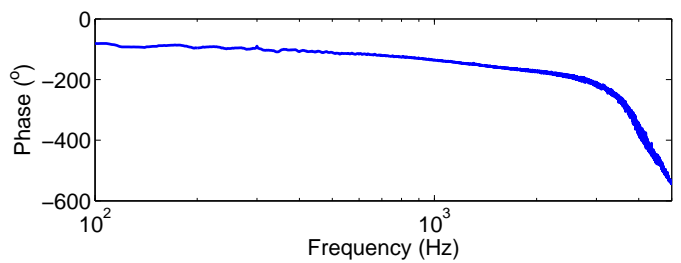

(b)

Figure 6: Frequency response of the PI controller in (a) and the open loop controller-actuator in the near-linear operating regime in (b).

$$
\begin{aligned}
\mathcal{H}= & \frac{1}{2}\left[(\mathbf{C x}(t)-r(t))^{T} Q(\mathbf{C} \mathbf{x}(t)-r(t))+u^{T}(t) R u(t)\right] \\
& +\lambda^{T}[\mathbf{A x}(t)+[\mathbf{B}(u)](t)+\mathbf{G}(t)]
\end{aligned}
$$

where penalties on the tracking error and the control input are given by the variables $Q$ and $R$, respectively.

The minimum of the cost functional in (25) is determined under the constraint of the differential equation given by (13). By employing Lagrange multipliers an unconstrained minimization problem is constructed where the stationary condition for the Hamiltonian yields the adjoint relation $[25,26]$

$$
\dot{\lambda}(t)=-\mathbf{A}^{\mathbf{T}} \lambda(t)-\mathbf{C}^{\mathbf{T}} Q \mathbf{C x}(t)+\mathbf{C}^{\mathbf{T}} Q r(t)
$$

and optimal control input

$$
u^{*}(t)=-R^{-1}\left(\frac{\partial \mathbf{B}(u)}{\partial u}\right)^{T} \lambda(t)
$$

The resulting optimality system is 

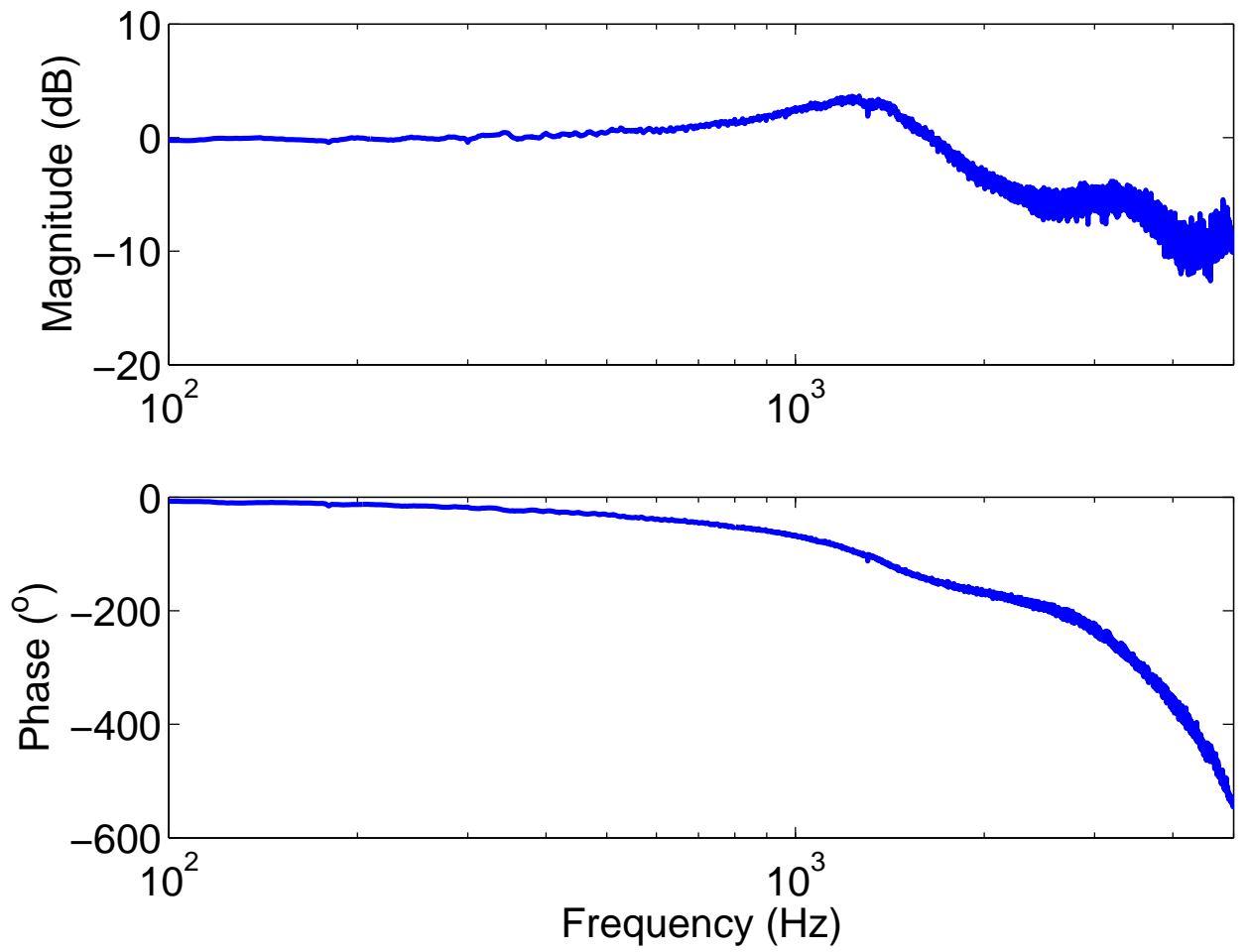

Figure 7: Frequency response of the closed loop controller-actuator system in the near linear operating regime.

$$
\begin{aligned}
& {\left[\begin{array}{c}
\dot{\mathbf{x}}(t) \\
\dot{\lambda}(t)
\end{array}\right]=\left[\begin{array}{c}
\mathbf{A} \mathbf{x}(t)+\left[\mathbf{B}\left(u^{*}\right)\right](t)+\mathbf{G}(t) \\
-\mathbf{A}^{\mathbf{T}} \lambda(t)-\mathbf{C}^{\mathbf{T}} Q \mathbf{C x}(t)+\mathbf{C}^{\mathbf{T}} Q r(t)
\end{array}\right]} \\
& \mathbf{x}\left(t_{0}\right)=\mathbf{x}_{\mathbf{0}} \\
& \lambda\left(t_{f}\right)=\mathbf{C}^{\mathbf{T}} P\left(\mathbf{C x}\left(t_{f}\right)-r\left(t_{f}\right)\right) .
\end{aligned}
$$

The force determined from (9) is included in the input operator $\left[\mathbf{B}\left(u^{*}\right)\right](t)$ which directly includes the rate-dependent nonlinear and hysteretic $H-M$ behavior within the control design. This dynamic system results in a two-point boundary value problem which precludes an efficient Ricatti formulation due to the nonlinear nature of the input operator. This system of equations and the boundary conditions are solved using a quasi-Newton method to determine the nonlinear magnetic field input; see [18] for more details. 
An additional step is necessary to implement the control design experimentally using the voltage control amplifier. As discussed in Section 3.3, the homogenized energy model can be used to quantify nonlinear inductance. This relation is used to numerically determine the voltage control from (15) since the current can be determined from the magnetic field in (28) and the current-field relation in (16).

The following steps are used to determine the voltage control input: 1) The optimal magnetic field is computed from (28). 2) The actuator is simulated by applying $u^{*}(t)$ to (13) to determine $\frac{d y}{d u^{*}}$ to obtain the nonlinear inductance. 3) The nonlinear voltage control is computed by solving the nonlinear ODE in (15) numerically. Numerical implementation of (15) uses a central difference approximation,

$$
V_{k}=\frac{1}{2}\left(L\left(M_{k}\right)+L\left(M_{k+1}\right)\right) \frac{i_{k+1}+i_{k}}{\Delta t}+\frac{1}{2} R\left(i_{k+1}+i_{k}\right)
$$

where a temporal step size $\Delta t$ is employed giving a discretization in time defined by $t_{k}=k \Delta t$. The voltage solved in (30) is the nonlinear open loop control input used in the experiments.

\subsubsection{Perturbation Feedback}

It is known that open loop controls are not robust with regard to operating uncertainties such as unmodeled constitutive behavior or disturbance loads. To mitigate these effects, PI perturbation feedback about the optimal open loop signal is implemented to improve robustness.

The perturbation control design is identical to the PI controller given in (24). The perturbation control can be written in the time domain as

$$
\delta u(t)=-K_{P} e(t)-K_{I} \int_{0}^{t} e(s) d s
$$

where $e(s)$ is the error between the measured displacement and the reference displacement. The perturbation control input is added to the system given by (13)

$$
\begin{aligned}
& \dot{\mathbf{x}}(t)=\mathbf{A} \mathbf{x}(t)+\left[\mathbf{B}\left(u^{*}+\delta u\right)\right](t) \\
& \mathbf{x}\left(t_{0}\right)=\mathbf{x}_{0}+\delta \mathbf{x}_{0}
\end{aligned}
$$


where the nonlinear input operator includes the optimal open loop control $u^{*}(t)$ and the perturbation feedback control $\delta u(t)$. Possible variations in the initial conditions are denoted by $\delta \mathbf{x}_{0}$. The control gains used in the perturbation feedback controller were the same as the PI controller presented in Section 4.1.

\section{Tracking Control Experimental Results}

The performance characteristics of the PI controller, open loop nonlinear optimal control design, and nonlinear optimal controller with PI perturbation feedback is given to illustrate operating regimes where the nonlinear controller is superior. The control experiments were initiated by a half-cycle $1 \mathrm{~Hz}$ sine wave with amplitude $1 \mathrm{~V}$. The reference displacement is taken to be zero at the onset of applying control; therefore, the initial remnant displacement occurring after the magnetization half cycle is subtracted from the total actuator displacement. The commanded reference input to the controller was a sinusoidal signal. The amplitude of the reference signal was chosen to be $30 \mu m$ which represents an operating regime where significant nonlinearities exist as previously illustrated in Figure 3. Control experiments for frequencies 100, 200,300,500,700, and $1000 \mathrm{~Hz}$ were conducted and the results are illustrated in Figure 8 for each control design. The length of the reference signal in all cases except for the $1 \mathrm{kHz}$ case was 4.25 periods. The $1000 \mathrm{~Hz}$ experiment was extended to 10 periods in order to observe the potential effects of drift using the open loop nonlinear optimal controller.

The comparison in tracking performance for each reference displacement signal is illustrated in Figure 8. Comparable performance was achieved between the PI controller and the nonlinear optimal control design for 100 and $200 \mathrm{~Hz}$ reference displacements. Marginal differences in tracking control between PI and the nonlinear control design become apparent at $300 \mathrm{~Hz}$ and at higher frequencies, the tracking error using PI control continues to increase. A phase lag occurs due to the hysteresis as the frequency increases above $500 \mathrm{~Hz}$. As the reference displacement frequency approaches the bandwidth of the PI controller, the amplification in the closed loop system previously shown in Figure 7 degrades tracking control; see Figure 8. This is also illustrated in Figure 10 where the voltage control input is shown for the $500 \mathrm{~Hz}$ and $1000 \mathrm{~Hz}$ cases. A phase lag is shown between the nonlinear open loop optimal control and the PI controller at $500 \mathrm{~Hz}$ while the PI controller over-amplifies the control signal at $1000 \mathrm{~Hz}$. Figure 10 illustrates how the nonlinear open loop control directly compensates for the 


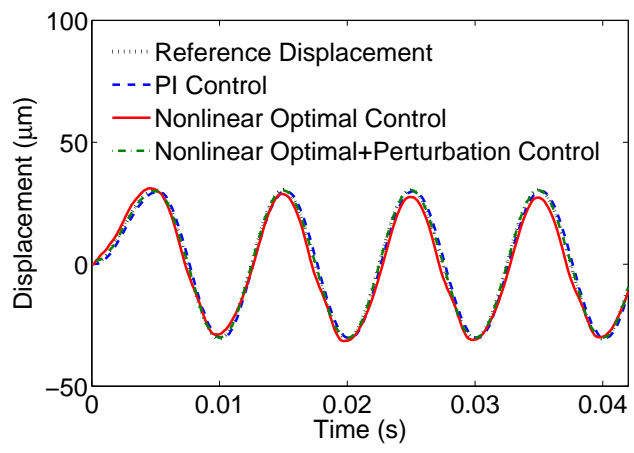

(a)

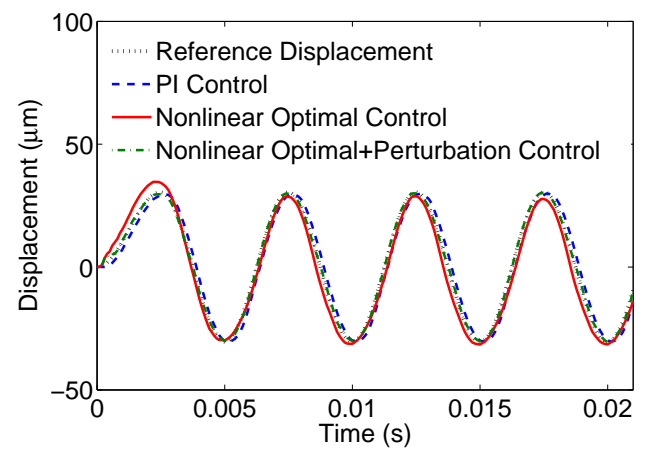

(c)

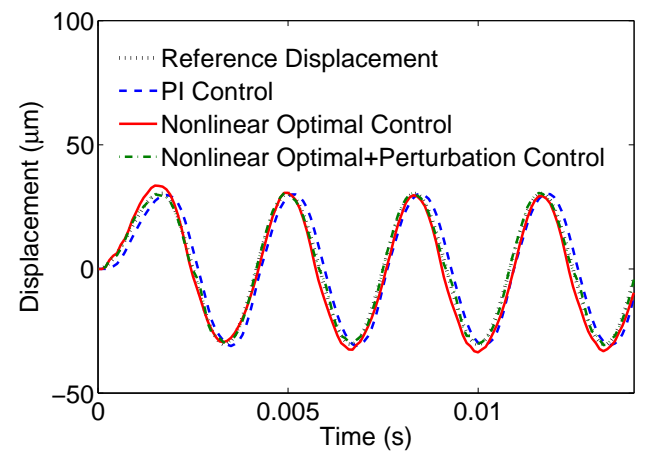

(e)

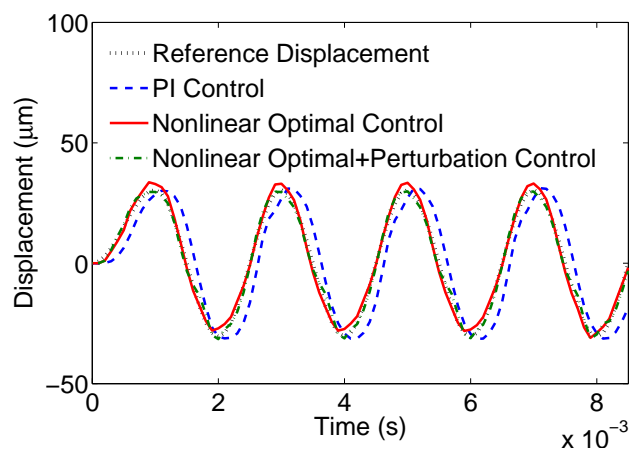

(b)

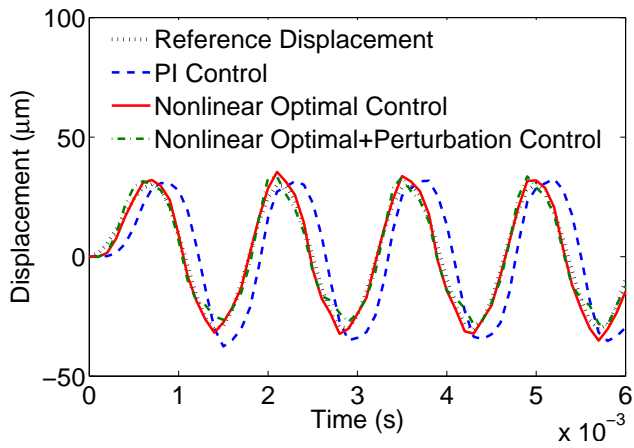

(d)

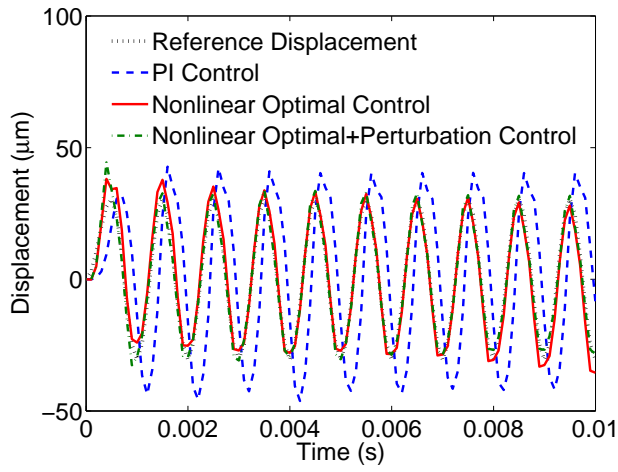

(f)

Figure 8: Comparison of the tracking control performance using PI control, nonlinear open loop optimal control and nonlinear open loop optimal control with PI perturbation feedback. The frequencies tested range from (a) $100 \mathrm{~Hz}$ (b) $200 \mathrm{~Hz}$ (c) $300 \mathrm{~Hz}$ (d) $500 \mathrm{~Hz}$ (e) $700 \mathrm{~Hz}$ (f) $1000 \mathrm{~Hz}$. 


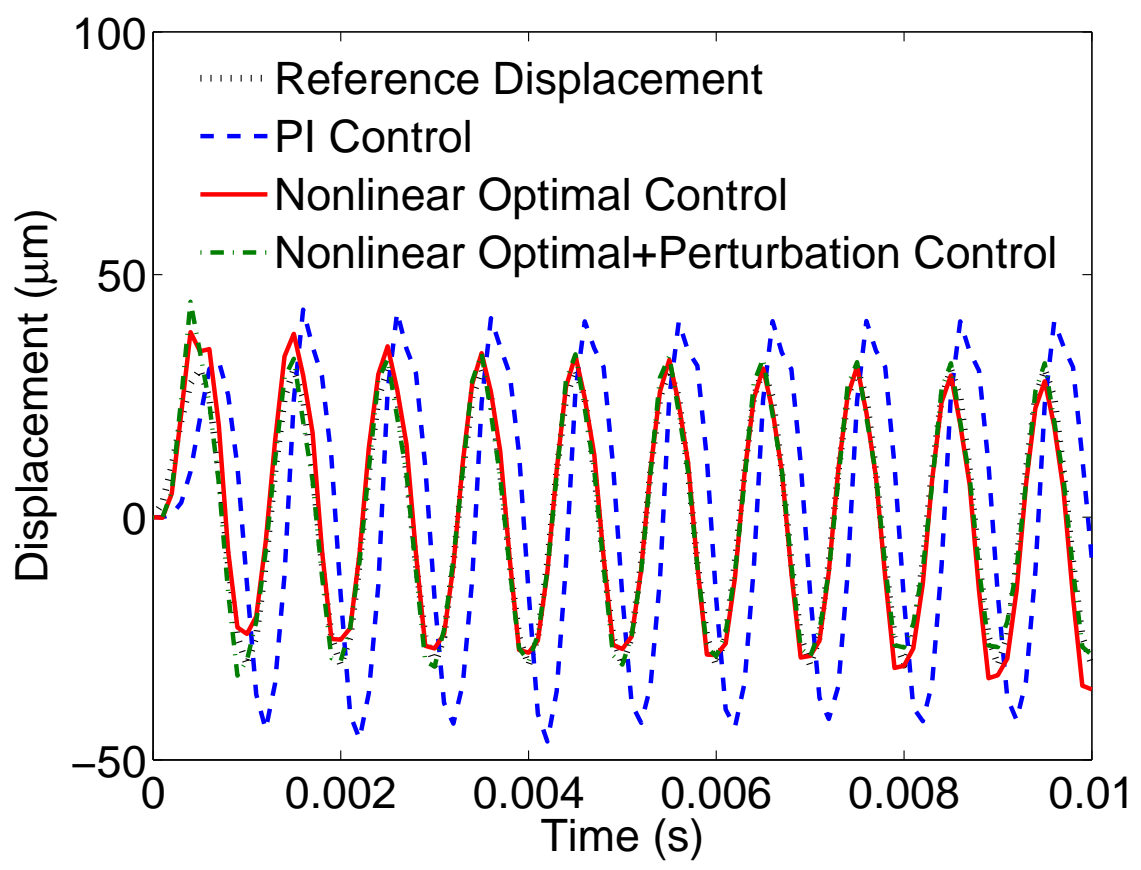

Figure 9: Tracking control experimental results at $1 \mathrm{kHz}$ expanded from Figure 8 to illustrate improvements in tracking control between PI control and nonlinear optimal control.

nonlinear and hysteretic constitutive behavior. It should also be noted that minor errors in drift occur at $1000 \mathrm{~Hz}$ using nonlinear open loop control, but this error is corrected by including perturbation feedback as shown in Figure 8(f) and expanded in Figure 9.

The tracking error for each experiment is quantified using the percent root-mean-square (RMS) error and is presented in Table 3. The percent RMS error is defined by

$$
e_{R M S}=\frac{\frac{1}{T} \int_{0}^{T} e^{2}(t) d t}{\max (y(t))-\min (y(t))}
$$

where $T$ is the final time in each experiment. A direct comparison of percent RMS error between nonlinear control with perturbation and PI control illustrate superior performance at all frequencies tested. The percent error was reduced by more than one order of magnitude at each frequency tested. It should also be noted that open loop nonlinear control and perturbation feedback control gave 


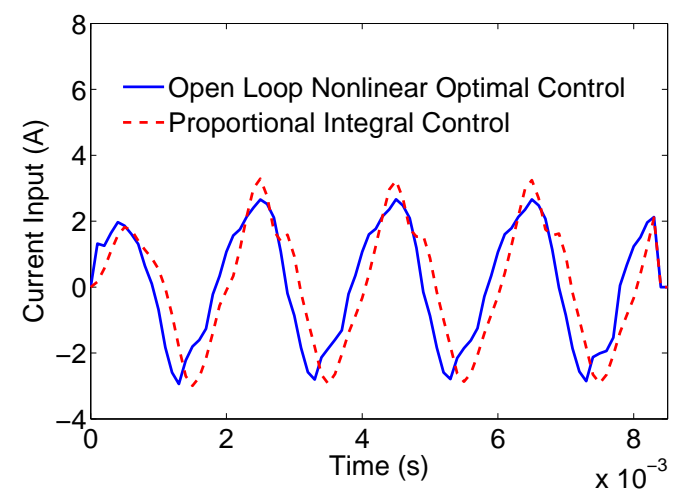

(a)

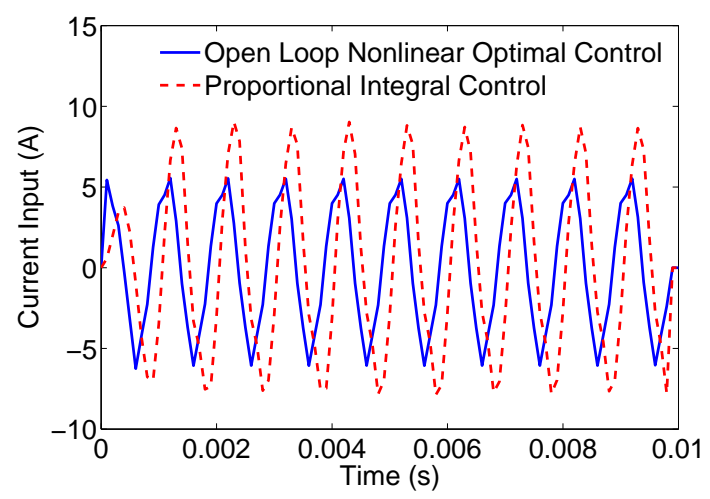

(b)

Figure 10: Comparison of current inputs using open loop nonlinear optimal control and PI control. (a) Current input for the $500 \mathrm{~Hz}$ reference displacement. (b) Current input for the $1000 \mathrm{~Hz}$ reference displacement.

approximately the same error at frequencies $\geq 500 \mathrm{~Hz}$. This is believed to be related to the bandwidth of the PI controller used for perturbation feedback since the control gains were the same as the PI control design.

\section{Discussion and Concluding Remarks}

The incorporation of the homogenized energy model in the nonlinear optimal control design was

Table 3: Percent RMS error for each tracking control experiment illustrated in Figure 8.

\begin{tabular}{l|l|l|l}
\hline Frequency & PI Control & Open Loop Optimal Control & Perturbation Control \\
\hline $100 \mathrm{~Hz}$ & $0.7 \%$ & $1.4 \%$ & $0.02 \%$ \\
$200 \mathrm{~Hz}$ & $2.3 \%$ & $2.2 \%$ & $0.08 \%$ \\
$300 \mathrm{~Hz}$ & $4.8 \%$ & $1.7 \%$ & $0.2 \%$ \\
$500 \mathrm{~Hz}$ & $15 \%$ & $0.73 \%$ & $0.8 \%$ \\
$700 \mathrm{~Hz}$ & $29 \%$ & $1.2 \%$ & $2.4 \%$ \\
$1000 \mathrm{~Hz}$ & $94 \%$ & $2.2 \%$ & $1.3 \%$ \\
\hline
\end{tabular}


shown to significantly improve tracking control at frequencies up to at least $1000 \mathrm{~Hz}$. Reasonable robustness in model predictions was illustrated by fitting a single set of rate-dependent material parameters to data between 100 and $500 \mathrm{~Hz}$ which was then used in controlling the Terfenol-D actuator up to $1 \mathrm{kHz}$. As previously noted, only current vs. displacement data was measured which required estimating the current vs. magnetization hysteresis loops. Since the induction of the magnetostrictive actuator was not measured, uncertainty in estimating certain rate-dependent model parameters occurred. While this limits achieving an accurate model prediction of both displacement and magnetization, it illustrates the ability to implement the model-based control design on magnetostrictive actuators in situations where the magnetization is not measurable but only displacement tracking is desired.

Previously, the homogenized energy model has focused on applying a magnetic field to quantify changes in the internal magnetization state. In the present analysis, the homogenized energy model was used to relate magnetic fields to voltage inputs. A simple relation was presented to relate the magnetic field in the Terfenol-D rod to the current in the wound wire solenoid. This approach assumed full penetration of the magnetic field for the frequency range considered which neglects eddy currents. The Terfenol-D rod was not laminated; therefore, the possibility of generating eddy currents is likely to occur in the frequency range tested. Further work is required to quantify this behavior and identify the appropriate method for including eddy currents in the model-based control design. The effect of ferromagetic switching behavior on the nonlinear inductance was included in the model, as previously illustrated in Figure 4. This gave improved model predictions, particularly in regions where more ferromagetic switching is expected to occur as shown in Figure 4, but approximating the inductance as linear may be sufficient depending on the performance requirements needed and the magnitude of magnetostrictive switching.

The nonlinear optimal control design has focused on applications where the reference displacement is known in advance and precise control is desired at relatively high speed. For these applications, the nonlinear control input can be computed off-line and then implemented in real-time control applications. Although the numerical procedure requires convergence of a nonlinear two-point boundary value problem, once the numerical procedure is developed, the control input for most reference signals (within physical limitations) can be quantified. This approach provides an alternative to nonlinear 
inverse compensator designs which depend on the ability to invert the constitutive model efficiently in real-time.

The PI controller provided good tracking control at 100 and $200 \mathrm{~Hz}$ and performance degradation began to occur at $300 \mathrm{~Hz}$. Amplification in the closed loop response (see Figure 7) increased the tracking error as the frequency approached the bandwidth of the system, see Figure 8(a-f). In addition, the effects of nonlinearities and hysteresis previously illustrated in Figure 3(d) become significant at higher frequencies which limits precise displacement control. Conversely, when the nonlinear optimal control design is implemented with perturbation feedback, the tracking errors are reduced and good performance is achieved up to $1000 \mathrm{~Hz}$. These experimental results used perturbation control gains that were identical to the PI control gains. Although larger gains were considered for perturbation control, reduction in tracking error was not achieved. Whereas the inclusion of open loop nonlinear control is expected to reduce the effect of phase lag from nonlinearities and hysteresis and allow the application of larger perturbation control gains, this was not the case. More work is required to identify this issue. Despite this effect, the hybrid nonlinear control design provides considerable improvements in bandwidth by including the homogenized energy model in the control design. Additionally, due to the general ferroic homogenized energy modeling framework, the control design can be potentially applied to a number of smart material systems and structures that use ferroelectric, magnetostrictive or shape memory alloys.

\section{Acknowledgments}

The authors gratefully acknowledge support from the Air Force Office of Scientific Research through grant AFOSR-FA9550-04-1-0203 and the Office of Naval Research through grant N000140610530. The authors also wish to acknowledge Dr. Julie Slaughter of Etrema Products, Inc., for supplying the Terfenol-D material used in this study.

\section{References}

[1] S.-E. Park and T. Shout, "Ultrahigh strain and piezoelectric behavior in relaxor based ferroelectric single crystals," J. Appl. Phys., vol. 82, no. 4, pp. 1804-18011, 1997. 
[2] F. Giessibl, "Advances in atomic force microscopy," Rev. Mod. Phys., vol. 75, pp. 949-983, 2003.

[3] J. Nealis and R. Smith, "Model-based robust control design for magnetostrictive transducers operating in hysteretic and nonlinear regimes," IEEE Trans. Control Syst. Technol., vol. 15, no. $1,2007$.

[4] C. Lynch, "The effect of uniaxial stress on the electro-mechanical response of 8/65/35 plzt," Acta. Mater., vol. 44, no. 10, pp. 4137-4148, 1996.

[5] R. O'Handley, S. Murray, M. Marioni, H. Nembach, and S. Allen, "Phenomenology and giant magnetic-field-induced strain in ferromagnetic shape-memory meterials," J. Appl. Phys., vol. 87, no. 9, pp. 4712-4717, 2000.

[6] R. Smith, M. Salapaka, A. Hatch, J. Smith, and T. De, "Model development and inverse compensator design for high speed nanopositioning," Proc. 41nd IEEE Conf. Decision Control, pp. 3652-3657, 2002.

[7] A. Cavallo, C. Natale, S. Pirozzi, C. Visone, and A. Formisano, "Feedback control systems for micropositioning tasks with hysteresis compensation," IEEE T. Magn., vol. 40, no. 2, 2004.

[8] X. Tan and J. Baras, "Modeling and control of hysteresis in magnetostrictive actuators," Automatica, vol. 40, pp. 1469-1480, 2004.

[9] P. Ge and M. Jouaneh, "Tracking control of a piezoceramic actuator," IEEE T. Contr. Syst. T., vol. 4, no. 3, pp. 209-216, 1996.

[10] H. Janocha and K. Kuhnen, "Real-time compensation of hysteresis and creep in piezoelectric actuators," Sensors and Actuators, vol. 79, pp. 83-89, 2000.

[11] S. Majima, K. Kodama, and T. Hasegawa, "Modeling of shape memory alloy actuator and tracking control system with the model," IEEE T. Contr. Syst. T., vol. 9, no. 1, pp. 54-59, 2001.

[12] W. Galinaitis and R. Rogers, "Compensation for hysteresis using bivariate preisach models," Proc. SPIE, Smart Struct. and Mater. 1997: Mathematics and Control in Smart Structures: San Diego, CA, 1997. 
[13] R. Smith, S. Seelecke, M. Dapino, and Z. Ounaies, "A unified framework for modeling hysteresis in ferroic materials," J. Mech. Phys. Solids, vol. 54, no. 1, pp. 46-55, 2005.

[14] R. Smith, M. Dapino, and S. Seelecke, "A free energy model for hysteresis in magnetostrictive transducers," J. Appl. Phys., vol. 93, no. 1, pp. 458-466, 2003.

[15] R. Smith, S. Seelecke, Z. Ounaies, and J. Smith, "A free energy model for hysteresis in ferroelectric materials," J. Intell. Mater. Syst. Struct., vol. 14, no. 11, pp. 719-739, 2003.

[16] R. Smith, A. Hatch, B. Mukherjee, and S. Liu, "A homogenized energy model for hysteresis in ferroelectric materials: General density formulations," J. Intell. Mater. Syst. Struct., vol. 16, no. 9, pp. 713-732, 2005.

[17] R. Smith, Smart Material Systems: Model Development. Philadelphia, PA: SIAM, 2005.

[18] R. Smith, "A nonlinear optimal control method for magnetostrictive actuators," J. Intell. Mater. Syst. Struct., vol. 9, no. 6, pp. 468-486, 1995.

[19] W. Oates and R. Smith, "Nonlinear optimal control techniques for vibration attenuation using nonlinear magnetostrictive actuators," to appear in the J. Intell. Mater. Sys. Struct., 2007.

[20] R. Smith, M. Dapino, T. Braun, and A. Mortensen, "A homogenized energy framework for ferromagnetic hysteresis," IEEE Trans. Mag., vol. 42, no. 7, pp. 1747-1769, 1995.

[21] T. Braun and R. Smith, "Efficient implementation algorithms for homogenized energy models," Continuum Mech. Thermodyn., vol. 18, no. 3-4, pp. 137-155, 2006.

[22] M. Dapino, R. Smith, and A. Flatau, "A structural strain model for magnetostrictive transducers," IEEE T. Magn., vol. 36, no. 3, pp. 545-556, 2000.

[23] R. Serway, Physics For Scientists and Engineers. Saunders College Publishing, 1990.

[24] W. Oates and R. Smith, "Nonlinear perturbation control for magnetic transducers," accepted to the IEEE Conference on Decision and Control, 2007. 
[25] A. Bryson and Y.-C. Ho, Applied Optimal Control. Waltham, MA: Blasidell Publishing Company, 1969.

[26] F. Lewis and V. Syrmos, Optimal Control. New York, NY: John Wiley and Sons, 1995. 